\title{
Estudio comparativo de la estabilidad lipídica de harinas de soja, sorgo, avena, salvado y germen de trigo con y sin extrusión
}

\author{
Comparative study of lipidic stability of soybean, sorghum, oat, \\ bran and wheat germ flours with and without extrusion
}

\begin{abstract}
Crosa, María José ${ }^{(1)}$, Curia, Ana ${ }^{(2)}$, Curutchet, Ana ${ }^{(3)}$, Cadenazzi, Mónica ${ }^{(4)}$, Dotta, Gabriela ${ }^{(5)}$, Ferreyra, Verónica ${ }^{(2)}$, $\overline{\text { Maidana, Fabianna }}^{(5)}$, Souto, Laura ${ }^{(1)}$, Escudero, Jorge ${ }^{(6)}$

(1) Gerencia de I+D+I, Laboratorio Tecnológico del Uruguay, LATU, Uruguay - ${ }^{(2)}$ Centro de Investigación y Desarrollo en Tecnologías para la Industrialización de Cereales y Oleaginosas del Instituto de Tecnología Industrial, INTI, Buenos Aires, Argentina - ${ }^{(3)}$ Departamento de Análisis de Productos Agropecuarios, Laboratorio Tecnológico del Uruguay, LATU, Uruguay - ${ }^{(4)}$ Consultor estadístico - ${ }^{(5)}$ Departamento de Cereales, Oleaginosos y Productos Derivados, Laboratorio Tecnológico del Uruguay, LATU, Uruguay - ${ }^{(6)}$ Cooperativa Agraria Nacional, Copagran.
\end{abstract}

Contacto: mcrosa@latu.org.uy

Recibido: 3/6/2013 - Aprobado: 1/11/2013

\begin{abstract}
Resumen
El consumo de harinas de grano entero está condicionado por la pérdida de calidad nutricional, funcional y sensorial durante su almacenamiento. En este estudio se evaluó el efecto del proceso de extrusión en la degradación lipídica de harina de varios tipos de granos y derivados (soja, sorgo, avena, salvado y germen de trigo). Se realizó seguimiento de ácidos grasos libres, humedad, actividad de agua y del desarrollo del descriptor "rancio-oxidado" por olfatación directa, en muestras de harina con y sin extrusión almacenadas durante 60 días en bolsa de polietileno de 30 micrones a 30-35 ${ }^{\circ} \mathrm{C}$. También se estudió la aceptabilidad de pan elaborado con $36 \%$ de incorporación de una combinación de harinas. Se realizaron medidas de color, materia grasa y actividad ureásica al inicio, medio y final del almacenamiento. Se ajustaron funciones de regresión de la diferencia "sin extrusión" y "con extrusión", para las medidas AGL, Aw, \%H. Los resultados obtenidos indicaron que la extrusión disminuye significativamente la degradación lipídica de las harinas de sorgo, salvado, soja, avena; sin embargo, la harina de germen extrudida tuvo un aumento de rancidez por oxidación. La incorporación de las harinas no extrudidas en la masa de pan disminuyó significativamente la aceptabilidad y afectó la textura y volumen del pan.

Palabras clave: Harinas compuestas, degradación, rancidez, hidrólisis, oxidación.
\end{abstract}

\begin{abstract}
$\underline{\text { Abstract }}$
Consumption of whole grain flour is determined by the loss of nutritional, functional and sensory quality during storage. This study evaluates the effect of the extrusion process in the lipid degradation of flour of various types of cereal (soybean, sorghum, oat bran and wheat germ). Free fatty acids, moisture, activity and the development of the "rancid-oxidized" descriptor were followed up by direct olfaction in flour samples with and without extrusion stored for 60 days in a polyethylene bag of 30 micron to (30-35) ${ }^{\circ} \mathrm{C}$. The acceptability of bread made with $36 \%$ incorporation of a combination of flours was also studied. Measurements of color, fat and urease activity at the beginning, middle and end of storage were conducted. Regression functions were fitted to the difference "without extrusion" and "extrusion" for measures of AGL, Aw, \% H. The results showed that extrusion significantly decreased lipid degradation of sorghum flour, bran, soybean, oat, however, the extruded germ meal had an increased oxidative rancidity. Incorporating flours without extruded bread dough decreased significantly the acceptability and affect the bread texture and volume.

Keywords: Multigrain flour, degradation, rancidity, hydrolysis, oxidation.
\end{abstract}

\section{Introducción}

Las harinas de grano entero se caracterizan por su aporte de fibra, vitaminas, minerales, antioxidantes y otros nutrientes que las diferencian de las harinas refinadas. La Organización Mundial de la Salud (Asamblea Mundial de la Salud, 2002) recomienda el aumento del consumo de fibra mediante la ingesta de "grano entero" para la prevención de enfermedades crónicas no transmisibles. El consumo de harinas de grano entero se ha relacionado con la reducción del riesgo de enfermedades cardíacas y diabetes e incluso puede ayudar al manejo del peso corporal y al retardo del proceso de envejecimiento (U.S. Department Of Agriculture y U.S. Department of Health and Human Services, 2010). La Asociación de Medicamentos y Alimentos de los Estados Unidos (U.S Food and Drug Administration, 2003) afirma: "Dietas ricas en alimentos de grano entero y otros alimentos de origen vegetal, y que son bajas en grasa total, en grasa saturada y colesterol, pueden reducir los riesgos de enfermedades cardíacas y ciertos tipos de cáncer". El servicio del Departamento de Agricultura de Estados Unidos (U.S. Department of Agriculture) en 2010 recomendaba que al 
menos el 50\% del consumo de grano debe ser grano entero; a partir de julio de 2014 la recomendación aumentará al 100\%.

La Organización de las Naciones Unidas para la Alimentación y la Agricultura (FAO) inició el "Programa de Harinas Compuestas" con el objeto de buscar nuevas posibilidades para la utilización de materias primas distintas y complementarias al trigo en la producción de pan, galletas, pastas y otros alimentos derivados de cereales. El término "harinas compuestas" se usa para indicar la mezcla de harinas de cereales y leguminosas, con aporte nutricional complementario. Estas harinas compuestas se están utilizando en diversas partes del mundo con el objeto de remediar, en parte, las deficiencias de proteínas, vitaminas y minerales de la dieta.

Su desarrollo comercial ha sido acotado por la vida útil más corta comparada con las harinas refinadas. El grano entero posee su contenido natural de grasas, aceites y de enzimas lipolíticas que dificultan su incorporación en los alimentos. Tait et al. (1988) estudiaron la disminución de la calidad del pan debido al enranciamiento de las harinas, resultados verificados por otros autores (Hansen y Rose, 1996; Pomeranz et al., 1992).

La degradación lipídica es la principal causa de reducción de la funcionalidad, palatabilidad y propiedades nutricionales. Las estrategias para estabilizar la harina de grano entero deberían estar focalizadas en el control de la actividad de las enzimas lipolíticas (Doblado et al., 2012). Vetrimani et al. (1990) sometieron el salvado a $175^{\circ} \mathrm{C}$ durante 40 minutos, logrando un aumento de la vida útil de 20 a 90 días. Rose et al. (2008) estudiaron la efectividad del tratamiento de calor, vapor y microondas en la disminución de la actividad de la lipasa, sin aumento de la oxidación.

La extrusión fue clasificada como una tecnología de cocción HTST (alta temperatura, corto tiempo), que provoca cambios químicos y físicos deseables en el alimento. La extrusión minimiza la degradación de los nutrientes por calor, mejora la digestibilidad por gelatinización del almidón y desnaturalización de la proteína, comparada con los procesos térmicos tradicionales. Es un proceso de alta productividad con una significativa retención de nutrientes. La extrusión destruye o inactiva los componentes antinutricionales o tóxicos (inhibidores de tripsina, hematoglutininas, etcétera), enzimas indeseables como la lipoxigenasa, peroxidasa y microorganismos y preserva las propiedades nutricionales (Sosa Moguel et al., 2009; Athar et al, 2006; Brennan et al., 2011; Killeit, 1994; Turgeon et al., 2011). El deterioro de las harinas no solo afecta su aporte nutricional, sino también la aceptabilidad sensorial del pan (Tait et al., 1988).

El objetivo de este trabajo es evaluar el efecto del proceso de extrusión en la degradación lipídica de harina de varios de tipos de granos y derivados (soja, sorgo, avena, salvado y germen de trigo).

\section{Materiales y Métodos}

\section{Análisis fisicoquímicos de las harinas}

Granulometría, tamizado en equipo Retsch, con Tamices ASTM \#18, \#60 y base, en amplitud 60 durante 10 minutos. Medida por triplicado.

Proteínas, expresada en gramos de proteína en $100 \mathrm{~g}$ de harina según AACC 46-30, método de combustión en equipo Leco FP-528

Fibra total e insoluble, expresada en gramos de fibra en $100 \mathrm{~g}$ de harina determinada por método gravimétrico enzimático según: Official Methods of Analysis of AOAC International método 985.29.

Cenizas, según ISO 2171.

Aw, medida en AQUALAB SERIES 3TE.

Humedad expresada en gramos de agua en $100 \mathrm{~g}$ de harina. En la harina de soja se siguió el protocolo de análisis basado en AOCS Bc
2-49. En el resto de las harinas, la determinación de humedad fue en base a la técnica AACC 44-40.

Materia grasa (\% MG), expresada en gramos de materia grasa en $100 \mathrm{~g}$ de materia seca de la harina. En equipo Soxtec de Tecator, solvente éter de petróleo ppa., se expresa en g/100 g de muestra de harina, luego de determinar el tiempo óptimo de extracción de materia grasa para cada harina. En soja basado en AOCS Bc 3-49.

Ácidos grasos libres (\% AGL), expresados en gramos de oleico en $100 \mathrm{~g}$ de materia grasa. Se determina por valoración de los ácidos grasos libres con solución de $\mathrm{NaOH}$.

Color, fue determinado en colorímetro HUNTERLAB PLUS XE. Las medidas de color fueron expresadas por los valores especiales obtenidos por el sistema CIE- $L^{*} a * b^{*}$; el parámetro $L^{*}$ describe la luminosidad en una escala de 0 (negro) a 100 (blanco puro), el parámetro $\mathrm{a}^{*}$ es matiz del color (+ rojo - verde), y el parámetro $\mathrm{b}^{*}$ es matiz del color (+ amarillo - azul). Se determinó el índice de blancura (WI), acorde a Hsu et al. (2003).

$$
\mathrm{WI}=100-\mathrm{RAIZ}((100-\mathrm{L}) *(100-\mathrm{L})+\mathrm{a} * \mathrm{a}+\mathrm{b} * \mathrm{~b})
$$

Actividad ureásica en soja, según AOCS Bc 9-58

\section{Evaluación sensorial del descriptor "rancio- oxidado"}

Un panel conformado por seis evaluadores seleccionados según la Norma IRAM 20005-1 evaluó las muestras por discusión y consenso en mesa redonda bajo la metodología de Análisis Descriptivo Cuantitativo (QDA). Los evaluadores fueron entrenados según las normativas: AOCS. 1989 y FIL-IDF 99C. 1997

Las unidades experimentales se evaluaron por olfatación directa. Las muestras se presentaron en vasos descartables de poliestireno con tapa codificadas con números de tres dígitos al azar. El descriptor evaluado fue rancio-oxidado. Aproximadamente $30 \mathrm{~g}$ de cada harina fueron servidos para cada evaluador. Se agitó el vaso previo a destaparlo y se realizaron aspiraciones cortas y profundas. Se evaluó en una escala lineal de $10 \mathrm{~cm}$ anclada en los extremos $(0=$ nada de intensidad de aroma rancio, $10=$ mucha intensidad de aroma rancio).

Para el entrenamiento en el descriptor crítico rancio-oxidado se empleó una muestra de aceite enranciado según la norma AOCS (1989), que estuvo disponible en las cuatro sesiones de evaluación. Se consensuó como valor de referencia de entrenamiento por aroma la muestra enranciada a $60^{\circ} \mathrm{C} / 14$ días como valor 6 en la escala sensorial de 0 a 10.

\section{Proceso de elaboración de las harinas}

Las harinas fueron elaboradas en la planta semi-industrial del Centro Cereales y Oleaginosas del Instituto Nacional de Tecnología Industrial ( 9 de Julio, Buenos Aires, Argentina). Las condiciones óptimas de procesamiento de cada tipo de harina se determinaron antes de este ensayo.

Para las harinas no extrudidas se procedió a la clasificación y limpieza de los granos usando una zaranda de "clasificación por tamaño", y luego se llevó a cabo la molienda de los mismos en un molino de martillo. Allí se usaron mallas de diferente luz para la obtención de la granulometría de las distintas harinas (soja, avena o sorgo). En el caso del salvado y germen de trigo se utilizaron tal como estaban.

En el caso de las harinas extrudidas, los granos clasificados y limpios fueron llevados hasta el tamaño de partícula inferior a $4 \mathrm{~mm}$ en un molino de martillo. Los granos molidos de avena, sorgo y soja, el germen y el salvado sin moler fueron procesados en una extrusora 
de tornillo simple de tres secciones con las siguientes especificaciones: longitud del barril, $200 \mathrm{~mm}$; diámetro del barril, $105 \mathrm{~mm}$; velocidad de tornillo de $60 \mathrm{rpm}$. La velocidad de alimentación de la extrusora fue en promedio $550 \mathrm{~kg} / \mathrm{h}$ y el tiempo de residencia del producto fue de tres segundos. La temperatura de extrusión fue puesta a punto para cada tipo de grano en ensayos previos.

En el procesamiento de la soja y el germen la torta obtenida luego de la extrusión se desgrasó parcialmente mediante prensa-tornillo. En la siguiente etapa se procedió al enfriado del producto mediante una corriente de aire que es forzada a pasar a través de la columna de secado. Este flujo de aire intercambia calor con el material molido, logrando de esta manera el enfriamiento y secado de las harinas. Finalmente se llevó a cabo la molienda final en molino de martillo hasta la granulometría deseada para cada harina. Todos los productos finales fueron envasados en bolsas de papel Kraft (doble lámina) con lámina interna de polietileno.

\section{Ensayos de almacenamiento de las harinas}

Seguimiento de degradación lipídica por hidrólisis. Se prepararon 10 muestras de $50 \mathrm{~g}$ en bolsas de polietileno de 30 micrones de cada tipo de harina. Se almacenaron en cámara acondicionada $35^{\circ} \mathrm{C}$, $65 \% \mathrm{HR}$, cada 10 días se tomaron muestras y se congelaron a $-18^{\circ} \mathrm{C}$ en envases con barrera de oxígeno y agua por un período no mayor a 10 días previo al análisis. Se analizaron: ácidos grasos libres, actividad de agua y humedad. En los días 0, 30 y 60 se realizaron duplicados de los muestreos, en los demás días el muestreo fue simple.

Se realizaron medidas de color y materia grasa. En la soja también se midió actividad ureásica. Los análisis se realizaron en los días cero (D0), treinta (D30) y sesenta (D60).

Seguimiento sensorial de degradación lipídica por oxidación. Una vez elaboradas todas las muestras fueron almacenadas en condición de freezer a $-18{ }^{\circ} \mathrm{C}$. Las unidades experimentales fueron ingresando de forma escalonada en la condición de estudio, de $30 \pm 2{ }^{\circ} \mathrm{C}$, de modo que coincidieran en una misma sesión de evaluación. La muestra " 0 " siempre fue mantenida en freezer. Los tiempos de almacenamiento fueron 20,40 y 60 días. Cada unidad experimental consistió en muestras de $200 \mathrm{~g}$ en bolsas de polietileno de 30 micrones, para cada tipo de harina. Se almacenaron en cámaras acondicionadas a $30 \pm 2{ }^{\circ} \mathrm{C}$.

\section{Ensayo de panificación}

Pretratamiento de harinas compuestas. Se tamizan las harinas de salvado, soja, avena, y sorgo sin extrusión, de manera de componer la misma granulometría que la harina extrudida y posteriormente se mezclan en diferentes proporciones logrando la siguiente composición centesimal: cenizas $(4,4 \%)$, humedad $(9,0 \%)$, fibra soluble $(1,1 \%)$, fibra insoluble $(29,5 \%)$, materia grasa $(5 \%)$, proteína $(24,5 \%)$. Previo a la elaboración del pan, la harina compuesta se pre-hidrató. Las harinas extrudidas se pre-hidrataron en relación 1:1 (harina: agua) en peso y las harinas no extrudidas se pre-hidrataron en relación 1:0,6 (harina: agua) en peso.

Proceso de elaboración de pan de molde. Se elaboró pan con harinas conservadas a temperatura de refrigeración de $5{ }^{\circ} \mathrm{C}$, durante 12 meses. Previo a la elaboración del pan se realizaron medidas de ácidos grasos, humedad y acidez de las harinas. La formulación del pan fue la siguiente: harina refinada (960 g), harina compuesta (540 g), agua de la prehidratación de la harina compuesta, agua (576 g), sal $(22,5 \mathrm{~g})$, aceite refinado de girasol con un $84,5 \%$ de ácido oleico $(45 \mathrm{~g})$, levadura deshidratada ( $21 \mathrm{~g})$, aditivo mejorador de pan compuesto por esteaoril lactilato de sodio, ácido ascórbico, azodicarbonamida, alfa amilasa fungal, pentosanas $(12 \mathrm{~g})$ y gluten vital $(60 \mathrm{~g})$. La harina compuesta se pre-hidrató antes de mezclar con los ingredientes sólidos en la amasadora. La harina compuesta extrudida se prehidrató con $540 \mathrm{~g}$ de agua. La harina compuesta no extrudida se prehidrató con $324 \mathrm{~g}$ de agua.

Se elaboró pan de molde según el siguiente procedimiento: se mezclaron los ingredientes secos menos la levadura en amasadora HS-30, se agregaron el agua y el aceite, se amasó a velocidad lenta durante $5 \mathrm{~min}$ y a velocidad rápida durante $2 \mathrm{~min}$. Se incorporó la levadura y se continuó amasando hasta que la masa quedara elástica. Se dejó descansar 5 a 10 min, se armaron bollos de 198 g, se dejaron descansar 15 min. Se arman y estiban en moldes con tapa de $60 \mathrm{~mm}$ de alto, $65 \mathrm{~mm}$ de ancho, $155 \mathrm{~mm}$ de largo. Se fermentaron durante 60 min a $35^{\circ} \mathrm{C}$ y $80 \%$ humedad. Se cocinaron a $200{ }^{\circ} \mathrm{C}$ durante $25 \mathrm{~min}$, se retiraron del horno y se desmoldaron inmediatamente. Se dejaron enfriar 2 horas.

Medida del índice de absorción de agua (IAA). La medida del índice de absorción en agua se realizó siguiendo el procedimiento de Anderson et al. (1969). Se pesaron 2,5 g de muestra en tubos de centrífuga de $50 \mathrm{~mL}$ y se le agregaron $30 \mathrm{~mL}$ de agua destilada, se termostatizó en baño de agua a $30{ }^{\circ} \mathrm{C}$ durante $30 \mathrm{~min}$, agitándolos cada $5 \mathrm{~min}$ en un vortex durante 15 segundos. Luego se centrifugó a temperatura ambiente, a $5000 \mathrm{rpm}$ por $30 \mathrm{~min}$. Se separó el sobrenadante y se pesó el gel. El IAA se registró como el peso del gel (g) /gramos de muestra. La medida se realizó por triplicado.

Medida del volumen del pan. La medida de volumen de pan se realizó por método de desplazamiento de semillas de canola. Se llenó totalmente un recipiente plástico de 2,0 L, con semillas de canola, se enrasó y se registró el peso de las semillas. Se colocó el pan en el recipiente, se completó con semillas y se volvió a enrasar. Se midió el volumen de las semillas que quedaron en la probeta y se registró ese volumen como el volumen del pan. La medida se realizó por triplicado.

Ensayo de TPA en pan de molde. Ensayo TPA (Texture Analysis Profile) con texturómetro TA.XT2 usando un cilindro de $36 \mathrm{~mm}$ con bordes redondeados, en las siguientes condiciones de medida: Test speed: $1 \mathrm{~mm} / \mathrm{sec}, 40 \%$ deformación en $2,43 \pm 0,02 \mathrm{~cm}$ (dos rodajas) y 5 segundos de relajación. Se realizaron 6 repeticiones en cada ensayo. Las muestras se cortaron en rebanadas de $2,5 \mathrm{~cm}$ de altura, la medida se realizó en el centro de la rebanada.

Evaluación sensorial del pan de molde. Las pruebas se llevaron a cabo en una sala de análisis estandarizada (ISO 8589:2007). Los consumidores (entre 40 y 50 por sesión) evaluaron la aceptabilidad de una rodaja de pan de $1 \mathrm{~cm}$, utilizando una escala hedónica estructurada de 9 puntos (1= Me disgusta muchísimo; $9=$ Me gusta muchísimo). Los evaluadores evaluaron una condición por vez en días diferentes y se utilizó agua sin gas como borrador.

\section{Diseño estadístico}

Estudio de la estabilidad lipídica de las harinas. Se realizó un diseño completamente aleatorizado (DCA) en siete momentos de muestreo en el caso de las medidas de degradación lipídica por hidrólisis y de cuatro momentos de muestreo en el caso de la degradación lipídica por oxidación. Fueron realizadas dos repeticiones reales en los días 0,30 y 60 en el seguimiento de degradación lipídica. Los factores de estudio son: "extrusión" con dos niveles (con y sin) y factor "harinas" con cinco niveles (soja, sorgo, avena, germen de trigo y salvado de trigo). Se ajustaron funciones de regresión según el comportamiento de las variables medidas en el período considerado.

Ensayo de panificación. Se realizó un diseño en bloques completamente aleatorizado (DBCA) con un factor de estudio "extrusión" con dos niveles (con y sin) y tres bloques. En cada bloque se compara una elaboración de pan con harinas con y sin extrusión. Se estudiaron los resultados de las variables medidas por ANOVA y se compararon por Tukey $5 \%$. 


\section{Resultados y Discusión}

\section{Análisis fisicoquímico de las harinas}

En la Tabla 1 se presentan los valores de caracterización nutricional de las harinas de soja, sorgo, avena, germen y salvado de trigo y su granulometría.

\begin{tabular}{|c|c|c|c|c|}
\hline Muestra & $\begin{array}{c}\% \\
\text { Cenizas }\end{array}$ & $\begin{array}{c}\% \\
\text { Fibra total }\end{array}$ & $\begin{array}{c}\% \\
\text { Proteína }\end{array}$ & $\begin{array}{c}\% \\
\text { Humedad }\end{array}$ \\
\hline Germen & 3,44 & 14,1 & 25,5 & 10,2 \\
\hline Soja & 5,8 & 27 & 41,6 & 8 \\
\hline Avena & 2,32 & 21,3 & 15,9 & 11,2 \\
\hline Salvado & 5,19 & 43,4 & 16 & 7,9 \\
\hline Sorgo & 0,9 & 4,1 & 7,4 & 13,8 \\
\hline
\end{tabular}

Tabla 1. Caracterización nutricional de las harinas de germen, soya, avena y salvado extrudidas.
El tamaño de partícula de las harinas extrudidas resultó ser levemente mayor que las no extrudidas, como se detalla en la Tabla 2.

En la Tabla 3 se presentan los valores de color, MG y actividad ureásica de las harinas con y sin extrusión. Se observó una disminución significativa del WI en las harinas de salvado y la soja con extrusión respecto a las sin extrusión. En el caso del salvado se observó un incremento del color rojo y en la soja aumentó el amarillo; además de la significativa disminución del parámetro $L^{*}$ en ambas harinas extrudidas.

El valor de actividad ureásica de la soja extrudida cumple con la normativa MERCOSUR para consumo humano; es 6,5 veces menor al de la harina no extrudida. Las harinas son parcialmente desgrasadas durante el proceso de extrusión. La harina de sorgo extrudida presenta un $51,5 \%$ menos de materia grasa, el salvado un $36,6 \%$, el germen un $26,6 \%$, la soja extrudida presenta solo un 5,7\% menos de grasa. La materia grasa de la avena extrudida no presenta diferencia con la no extrudida.

\begin{tabular}{|c|c|c|c|c|c|c|}
\hline & & $\begin{array}{c}\text { Tamiz } \mathbf{1 8} \\
\text { (malla } 1 \mathrm{~mm} \text { ) }\end{array}$ & $\begin{array}{c}\text { Tamiz 40 } \\
\text { (malla } 0,42 \mathrm{~mm} \text { ) }\end{array}$ & $\begin{array}{c}\text { Tamiz 60 } \\
\text { (malla } 0,25 \mathrm{~mm} \text { ) }\end{array}$ & $\begin{array}{c}\text { Tamiz } 80 \\
\text { (malla } 0,177 \mathrm{~mm} \text { ) }\end{array}$ & Base \\
\hline \multirow{2}{*}{ SALVADO } & S/Extrusión & $25,4 \%$ & & $70,7 \%$ & & $3,8 \%$ \\
\hline & C/Extrusión & $50,4 \%$ & & $42,0 \%$ & & $7,6 \%$ \\
\hline \multirow{2}{*}{ GERMEN } & S/Extrusión & $38,5 \%$ & & $57,5 \%$ & & \\
\hline & $\mathrm{C} /$ Extrusión & $38,0 \%$ & & $50,4 \%$ & & $11,6 \%$ \\
\hline \multirow{2}{*}{ SOJA } & S/Extrusión & & $13,1 \%$ & & $84,3 \%$ & $2,0 \%$ \\
\hline & $\mathrm{C} /$ Extrusión & & $44,0 \%$ & & $47,5 \%$ & $8,5 \%$ \\
\hline \multirow{2}{*}{ AVENA } & S/Extrusión & $32,3 \%$ & & $52,6 \%$ & & $15,3 \%$ \\
\hline & C/Extrusión & $0,0 \%$ & $63,5 \%$ & & & $36,5 \%$ \\
\hline \multirow{2}{*}{ SORGO } & S/Extrusión & $31,7 \%$ & & $60,6 \%$ & & $7,7 \%$ \\
\hline & C/Extrusión & $0,05 \%$ & & $76,46 \%$ & & $26,49 \%$ \\
\hline
\end{tabular}

Tabla 2. Granulometría de las cinco harinas extrudidas.

\begin{tabular}{|c|c|c|c|c|c|c|c|c|c|c|c|c|c|}
\hline & & \multicolumn{2}{|c|}{$\mathbf{L}$} & \multicolumn{2}{|c|}{ a } & \multicolumn{2}{|c|}{ b } & \multicolumn{2}{|c|}{ WI } & \multicolumn{2}{|c|}{$\begin{array}{c}\% \text { Materia Grasa } \\
\text { (Bs.) }\end{array}$} & \multicolumn{2}{|c|}{$\begin{array}{c}\text { Actividad ureásica } \\
\text { (Bs.) }\end{array}$} \\
\hline & & Media & $\begin{array}{c}\text { P valor } \\
\text { DMS }\end{array}$ & Media & $\begin{array}{c}\text { Pvalor } \\
\text { DMS }\end{array}$ & Media & $\begin{array}{c}\text { P valor } \\
\text { DMS }\end{array}$ & Media & $\begin{array}{c}\text { P valor } \\
\text { DMS }\end{array}$ & Media & $\begin{array}{l}\text { P valor } \\
\text { DMS }\end{array}$ & Media & $\begin{array}{c}\text { P valor } \\
\text { DMS }\end{array}$ \\
\hline \multirow{2}{*}{ SALVADO } & S/Extrusión & $54,67 \mathrm{a}$ & \multirow{2}{*}{$\begin{array}{c}\mathbf{0 , 0 1 1} \\
4,53\end{array}$} & $7,76 \mathrm{a}$ & \multirow{2}{*}{$\begin{array}{c}\mathbf{0 , 0 2 5} \\
0,78\end{array}$} & $14,06 \mathrm{a}$ & \multirow{2}{*}{$\begin{array}{r}\mathbf{0 , 3 1 3} \\
1,32\end{array}$} & 51,91 a & 0,01 & $3,33 \mathrm{a}$ & \multirow{2}{*}{$\begin{array}{l}\mathbf{0 , 0 1} \\
0,57\end{array}$} & & \\
\hline & C/Extrusión & $47,46 \mathrm{~b}$ & & $6,78 \mathrm{~b}$ & & $14,61 \mathrm{a}$ & & $45,04 \mathrm{~b}$ & 4,32 & $2,11 \mathrm{~b}$ & & & \\
\hline \multirow{2}{*}{ GERMEN } & S/Extrusión & $62,03 \mathrm{a}$ & \multirow{2}{*}{$\begin{array}{c}\mathbf{0 , 1 0 7} \\
2,14\end{array}$} & $6,06 \mathrm{a}$ & \multirow{2}{*}{$\begin{array}{c}\mathbf{0 , 0 0 0 4} \\
0,56\end{array}$} & $16,92 \mathrm{a}$ & \multirow{2}{*}{$\begin{array}{c}\mathbf{0 , 9 9 3} \\
2,03\end{array}$} & $57,98 \mathrm{a}$ & 0,26 & 9,05 a & \multirow{2}{*}{$\begin{array}{c}\mathbf{0 , 0 0 2} \\
0,95\end{array}$} & & \\
\hline & C/Extrusión & $60,43 \mathrm{a}$ & & $3,82 \mathrm{~b}$ & & $16,92 \mathrm{a}$ & & $56,80 \mathrm{a}$ & 2,54 & $6,68 \mathrm{~b}$ & & & \\
\hline \multirow{2}{*}{ SOJA } & S/Extrusión & $72,95 \mathrm{~b}$ & \multirow{2}{*}{$\begin{array}{c}\mathbf{0 , 0 1 7} \\
1,31\end{array}$} & $3,14 \mathrm{a}$ & \multirow{2}{*}{$\begin{array}{c}\mathbf{0 , 0 7 2} \\
1,79\end{array}$} & $21,19 \mathrm{~b}$ & \multirow{2}{*}{$\begin{array}{c}\mathbf{0 , 0 0 1 5} \\
0,87\end{array}$} & $65,72 \mathrm{a}$ & 0,0023 & $11,11 \mathrm{a}$ & \multirow{2}{*}{$\begin{array}{c}\mathbf{0 , 0 0 0 3} \\
0,16\end{array}$} & $1,82 \mathrm{~b}$ & \multirow{2}{*}{$\begin{array}{c}<\mathbf{0 , 0 0 0 1} \\
0,48\end{array}$} \\
\hline & C/Extrusión & $73,26 \mathrm{a}$ & & $1,57 \mathrm{a}$ & & $23,62 \mathrm{a}$ & & $62,87 \mathrm{~b}$ & 1,15 & $10,47 \mathrm{~b}$ & & $0,27 \mathrm{a}$ & \\
\hline \multirow{2}{*}{ AVENA } & S/Extrusión & $73,8 \mathrm{a}$ & \multirow{2}{*}{$\begin{array}{c}\mathbf{0 , 1 0 1} \\
4,38\end{array}$} & $3,46 \mathrm{a}$ & 0,852 & $13,12 \mathrm{a}$ & \multirow{2}{*}{$\begin{array}{c}\mathbf{0 , 0 5 4} \\
1,91\end{array}$} & $66,67 \mathrm{a}$ & 0,09 & $5,05 \mathrm{a}$ & \multirow{2}{*}{$\begin{array}{l}\mathbf{0 , 9 6} \\
1,86\end{array}$} & & \\
\hline & C/Extrusión & $70,4 \mathrm{a}$ & & $3,39 \mathrm{a}$ & 1,07 & $14,98 \mathrm{a}$ & & $70,46 \mathrm{a}$ & 4,76 & $5,09 \mathrm{a}$ & & & \\
\hline \multirow{2}{*}{ SORGO } & S/Extrusión & $77,43 \mathrm{a}$ & 0,71 & $2,00 \mathrm{a}$ & 0,027 & 13,94 a & 0,223 & $73,40 \mathrm{a}$ & 0,53 & $1,73 \mathrm{a}$ & \multirow{2}{*}{$\begin{array}{c}<\mathbf{0 , 0 0 0 1} \\
0,05\end{array}$} & & \\
\hline & C/Extrusión & $77,82 \mathrm{a}$ & 2,67 & $1,52 \mathrm{~b}$ & 0,39 & $13,17 \mathrm{a}$ & 1,49 & $74,16 \mathrm{a}$ & 3,05 & $0,84 \mathrm{~b}$ & & & \\
\hline
\end{tabular}

Tabla 3. Valores de color, \% materia grasa y actividad ureásica de las harinas con y sin extrusión. Letras iguales entre valores para un mismo tipo de harina indican que no existen diferencias significativas entre los procesos, según Tukey al $5 \%$. 


\section{Seguimiento de degradación lipídica por hidrólisis}

En la Tabla 4 se presentan los valores promedios de AGL, Aw y \%H de las harinas con y sin extrusión.

\begin{tabular}{|c|c|c|c|c|c|c|c|}
\hline \multirow[b]{2}{*}{ DÍAS } & \multirow[b]{2}{*}{ TRAT } & \multicolumn{3}{|c|}{ EXTRUDIDO } & \multicolumn{3}{|c|}{ NO EXTRUDIDO } \\
\hline & & $\%$ AGL & AW & HUMEDAD & $\%$ AGL & AW & HUMEDAD \\
\hline o & SALVADO & 1,49 & 0,520 & 8,1 & 28,67 & 0,700 & 14,0 \\
\hline 10 & SALVADO & 16,20 & 0,569 & 8,4 & 36,39 & 0,686 & 12,9 \\
\hline 20 & SALVADO & 14,50 & 0,566 & 8,8 & 33,33 & 0,660 & 12,1 \\
\hline 30 & SALVADO & 13,85 & 0,590 & 8,4 & 36,85 & 0,600 & 12,1 \\
\hline 40 & SALVADO & 12,40 & 0,526 & 9,1 & 37,20 & 0,636 & 11,8 \\
\hline 50 & SALVADO & 14,80 & 0,540 & 9 & 48,10 & 0,588 & 11,4 \\
\hline 60 & SALVADO & 14,85 & 0,537 & 8,9 & 55,35 & 0,500 & 10,1 \\
\hline $\mathbf{0}$ & GERMEN & 6,48 & 0,640 & 10,5 & 14,16 & 0,700 & 2,9 \\
\hline 10 & GERMEN & 5,75 & 0,625 & 9,5 & 25,94 & 0,685 & 12,5 \\
\hline 20 & GERMEN & 6,23 & 0,613 & 9,5 & 30,26 & 0,662 & 12,1 \\
\hline 30 & GERMEN & 6,25 & 0,618 & 9 & 32,20 & 0,700 & 11,8 \\
\hline 40 & GERMEN & 6,60 & 0,596 & 9,5 & 36,90 & 0,636 & 11,5 \\
\hline 50 & GERMEN & 7,70 & 0,592 & 9,3 & 37,70 & 0,596 & 10,6 \\
\hline 60 & GERMEN & 7,85 & 0,550 & 8,3 & 40,95 & 0,600 & 10,1 \\
\hline 0 & SOJA & 1,47 & 0,519 & 8,1 & 4,39 & 0,600 & 10,1 \\
\hline 10 & SOJA & 1,43 & 0,578 & 9,1 & 5,54 & 0,626 & 9,7 \\
\hline 20 & SOJA & 1,72 & 0,551 & 8,8 & 6,93 & 0,630 & 10,1 \\
\hline 30 & SOJA & 1,20 & 0,570 & 8,8 & 8,55 & 0,600 & 10,3 \\
\hline 40 & SOJA & 1,40 & 0,533 & 8,7 & 8,40 & 0,622 & 10,1 \\
\hline 50 & SOJA & 1,40 & 0,550 & 8,8 & 10,60 & 0,617 & 10,1 \\
\hline 60 & SOJA & 1,55 & 0,561 & 8,8 & 11,85 & 0,600 & 9,5 \\
\hline 0 & AVENA & 5,69 & 0,634 & 11,2 & 38,91 & 0,600 & 11,0 \\
\hline 10 & AVENA & 5,06 & 0,623 & 11,1 & 49,22 & 0,585 & 10,6 \\
\hline 20 & AVENA & 5,74 & 0,606 & 10,9 & 58,71 & 0,561 & 10,9 \\
\hline 30 & AVENA & 4,10 & 0,605 & 10,3 & 61,60 & 0,600 & 10,9 \\
\hline 40 & AVENA & 4,00 & 0,559 & 11 & 66,20 & 0,550 & 11,0 \\
\hline 50 & AVENA & 5,30 & 0,542 & 10,6 & 68,50 & 0,522 & 10,3 \\
\hline 60 & AVENA & 5,30 & 0,503 & 10,1 & 70,65 & 0,500 & 10,2 \\
\hline 0 & SORGO & 23,09 & 0,725 & 13,8 & 31,50 & 0,700 & 13,8 \\
\hline 10 & SORGO & 23,27 & 0,672 & 13,2 & 33,60 & 0,666 & 13,1 \\
\hline 20 & SORGO & 19,31 & 0,609 & 12,6 & 45,98 & 0,618 & 12,5 \\
\hline 30 & SORGO & 17,45 & 0,637 & 12,7 & 52,90 & 0,600 & 12,6 \\
\hline 40 & SORGO & 16,00 & 0,596 & 12,9 & 59,70 & 0,562 & 12,6 \\
\hline 50 & SORGO & 20,20 & 0,534 & 12,1 & 55,40 & 0,561 & 11,8 \\
\hline 60 & SORGO & 21,55 & 0,500 & 11,2 & 60,50 & 0,500 & 11,5 \\
\hline
\end{tabular}

Tabla 4. Valores de ácidos grasos calculados en base seca (AGL BS), actividad de agua (AW), humedad, durante el almacenamiento a $35 \pm$ $2{ }^{\circ} \mathrm{C}$ en las harinas. Los días 0,30 y 60 los valores corresponden al promedio de dos repeticiones reales.

El efecto del proceso de extrusión en las variables AGL, Aw y \% humedad durante el período de almacenamiento se determinó mediante el ajuste de regresión lineal simple de la diferencia entre los valores de la harina sin extrudir y de la harina extrudida. En la Tabla 5 se observan los resultados del ajuste. 


\begin{tabular}{|c|c|c|c|c|c|c|c|}
\hline & \multicolumn{7}{|c|}{ AGL (g ácidos libres / g sólidos secos) } \\
\hline & \multicolumn{3}{|c|}{$\beta_{0}$} & \multicolumn{4}{|c|}{$\beta_{1}$} \\
\hline & Media & $\mathbf{L I}_{95 \%}$ & $\mathbf{L S}_{95 \%}$ & Media & $\mathbf{L I}_{95 \%}$ & $\mathbf{L S}_{95 \%}$ & P valor \\
\hline Avena & 37,81 & 32,56 & 43,07 & 0,52 & 0,38 & 0,66 & 0,0001 \\
\hline Germen & 12,14 & 7,51 & 16,77 & 0,39 & 0,26 & 0,51 & 0,0001 \\
\hline Salvado & 20,59 & 13,11 & 28,08 & 0,24 & 0,14 & 0,45 & 0,05 \\
\hline Soja & 2,98 & 2,31 & 3,65 & 0,12 & 0,10 & 0,14 & 0,0001 \\
\hline \multirow[t]{2}{*}{ Sorgo } & 11,84 & 3,01 & 20,66 & 0,54 & 0,30 & 0,78 & 0,0008 \\
\hline & \multicolumn{7}{|c|}{ Aw } \\
\hline Avena & $-0,04$ & $-0,06$ & $-0,03$ & 0,00041 & 0,00008 & 0,0007 & 0,02 \\
\hline Germen & 0,06 & 0,05 & 0,07 & $-0,00067$ & $-0,0003$ & $-0,003$ & 0,0017 \\
\hline Salvado & 0,18 & $-0,005$ & $-0,002$ & $-0,0034$ & $-0,005$ & $-0,002$ & 0,0004 \\
\hline Soja & 0,09 & 0,07 & 0,11 & $-0,0008$ & $-0,0001$ & $-0,001$ & 0,02 \\
\hline \multirow[t]{2}{*}{ Sorgo } & $-0,02$ & $-0,05$ & 0,001 & 0,0005 & $-0,00009$ & 0,001 & --- \\
\hline & \multicolumn{7}{|c|}{ Humedad (g agua/g sólido húmedo) } \\
\hline Avena & $-0,16$ & $-0,72$ & $+0,40$ & 0,01 & $-0,01$ & 0,02 & --- \\
\hline Germen & $-3,13$ & $-7,53$ & $+1,28$ & 0,11 & $-0,01$ & 0,23 & --- \\
\hline Salvado & 5,59 & 5,05 & 6,13 & $-0,07$ & $-0,09$ & $-0,06$ & 0,0001 \\
\hline Soja & 1,70 & 1,18 & 2,23 & $-0,01$ & $-0,03$ & 0,001 & 0,07 \\
\hline Sorgo & $-0,13$ & $-0,51$ & $+0,25$ & 0,003 & $-0,01$ & 0,01 & --- \\
\hline
\end{tabular}

Tabla 5. Resultados del ajuste de la diferencia de cada variable entre las harinas sin extrudir y extrudida por regresión lineal simple, y $=\beta 0$ $+\beta 1 x$. Siendo " $y$ " el valor de la diferencia y " $x$ " el tiempo en días del ensayo. Se informa el valor medio del coeficiente de regresión (Coef. Reg.), el límite inferior ( $\mathrm{LI}_{95 \%}$ ) y el límite superior ( $\mathrm{LS}_{95 \%}$ ) del intervalo de confianza calculado con un $95 \%$ de confianza. El p-valor indica la probabilidad de que el coeficiente de regresión $(\beta 1)$ no sea significativamente distinto de cero.

La diferencia en el contenido de ácidos grasos libres presentó una ordenada en el origen y un coeficiente de regresión positivo y significativo para todas las harinas evaluadas. La diferencia entre las harinas aumenta durante el almacenamiento. La coordenada en el origen de la avena fue significativamente mayor respecto a las otras harinas. Le siguen de mayor a menor los valores de ordenadas en el origen del salvado, germen, sorgo y soja. Los valores de pendientes del sorgo, avena, salvado y germen no presentan diferencia significativa entre ellos y son significativamente mayores a la pendiente de la soja.

El ajuste por regresión lineal de la diferencia entre harina con y sin extrusión de las variables Aw y \% humedad si bien en algunas harinas fue significativo, en todos los casos los valores de ordenada en el origen y coeficientes de regresión fueron muy cercanos a cero e indicando una diferencia muy pequeña entre las harinas durante el almacenamiento.

\section{Seguimiento de degradación lipídica por oxidación}

A continuación se expresan en gráficos los valores medios del descriptor "rancio-oxidado" de cada harina con y sin extrusión. Los valores se ajustaron por regresiones logísticas para variables continuas con todos los datos del panel. El análisis de varianza de la regresión ajustada resultó significativo para todas las harinas con y sin extrusión (al 5\% de nivel de significación). El modelo del ajuste logístico aplicado se presenta a continuación:

$$
y=A+\frac{C}{1+e^{\left[-B^{*}(x-M)\right]}}
$$

Donde $\mathrm{x}$ es el tiempo, y la intensidad de aroma rancio y A, B, $\mathrm{C}$ y M son los parámetros de la regresión logística. Las regresiones ajustadas con sus promedios se presentan en los Gráficos 1 a 5 .

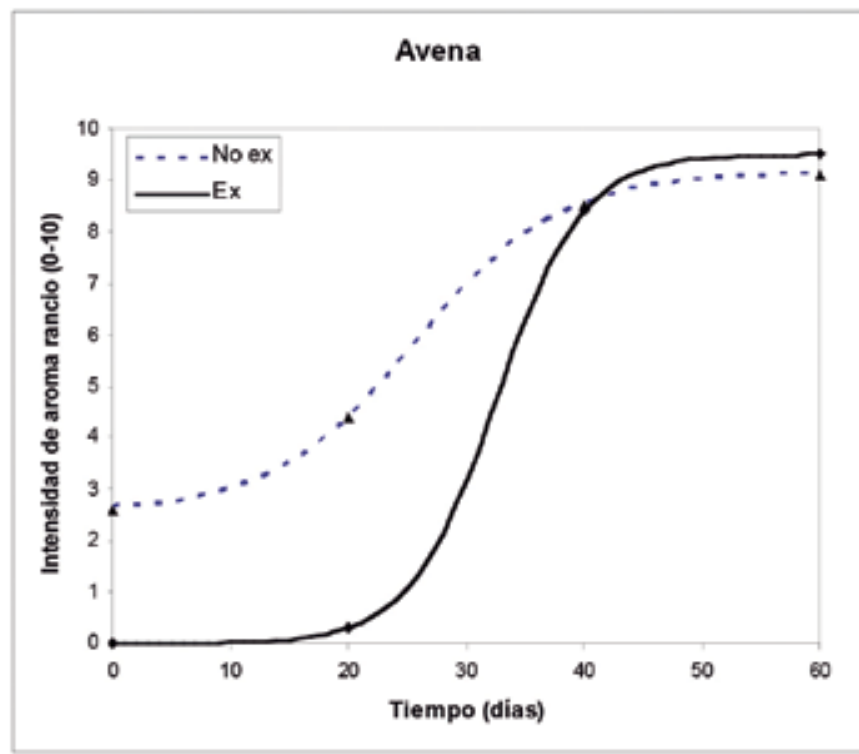




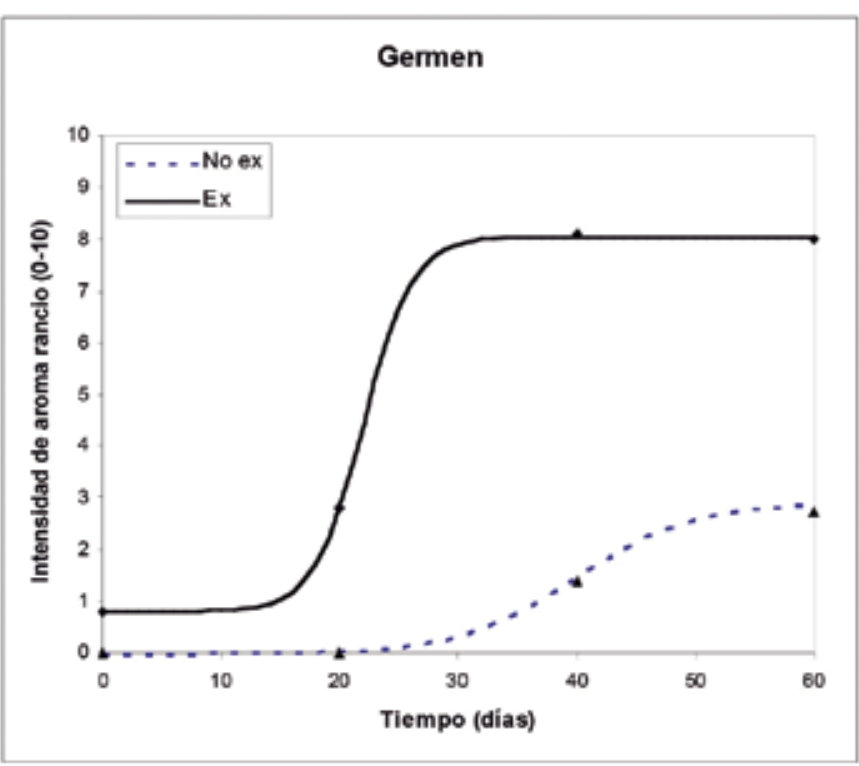

Gráfico 2. Regresión logística germen.

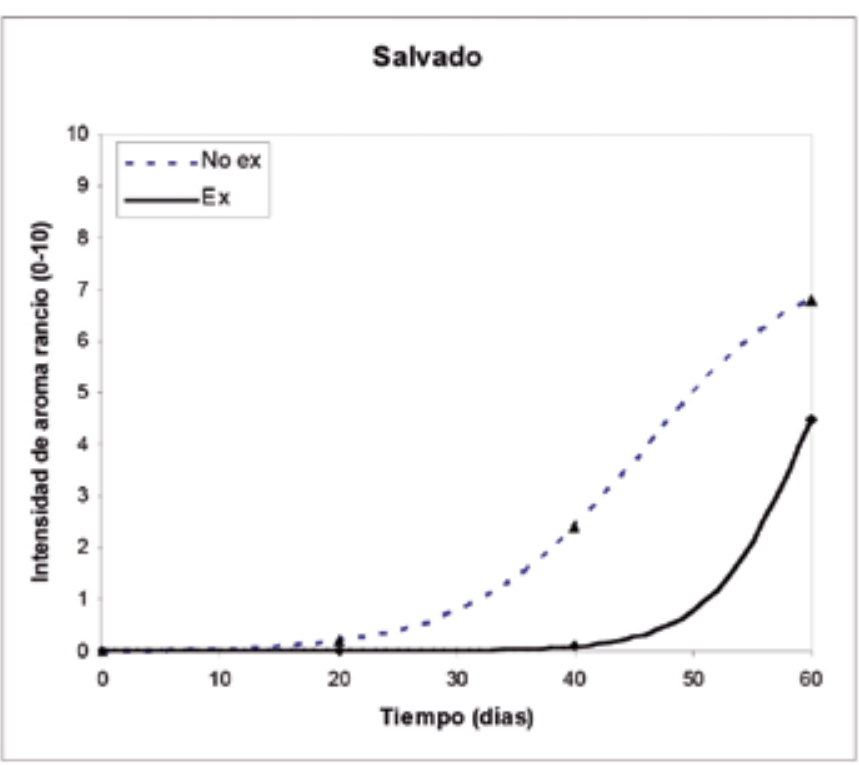

Gráfico 4. Regresión logística salvado.

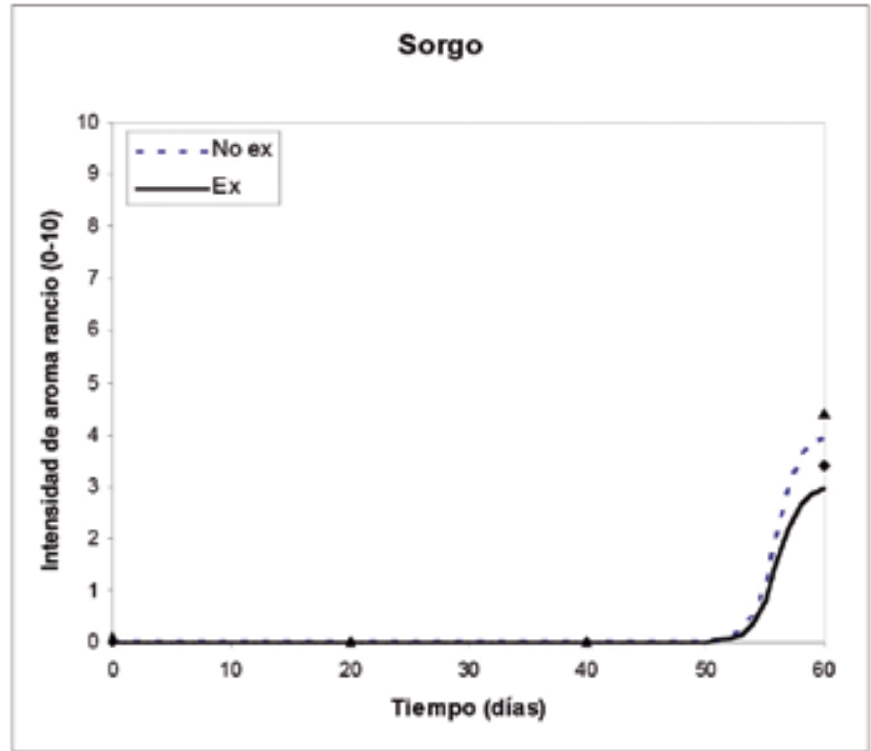

Gráfico 3. Regresión logística sorgo.

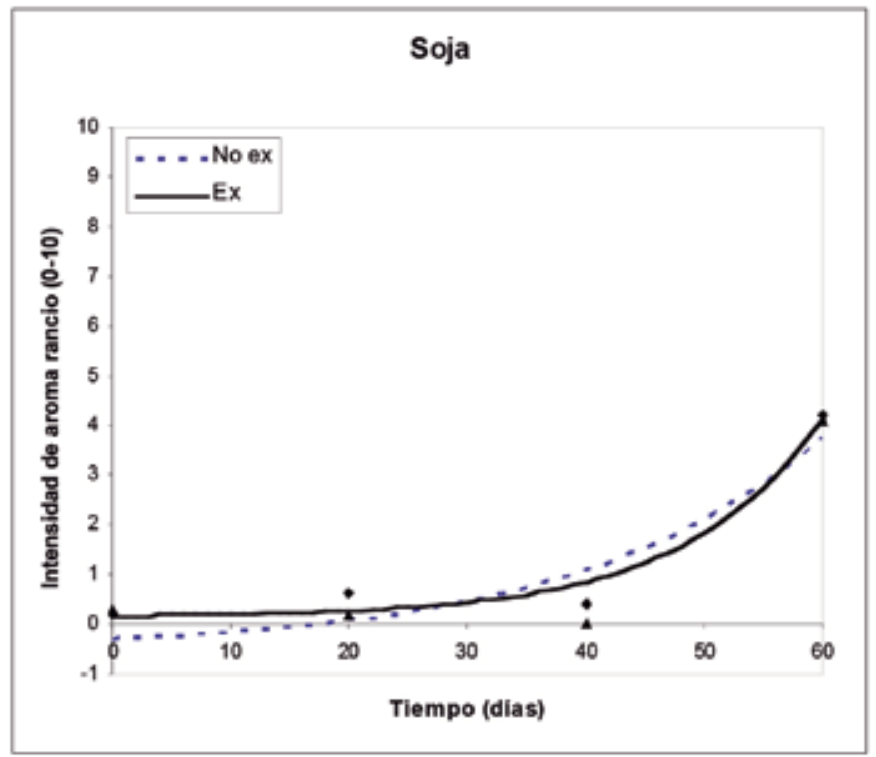

Gráfico 5. Regresión logística soja.

En la harina de avena y en el salvado el tratamiento de extrusión tiene un efecto positivo a fin de evitar el enranciamiento sensorial. La harina de avena no extrudida en el tiempo 0 ya presenta una intensidad apreciable de aroma rancio, lo que posiblemente se deba a que el freezado de las muestras atenuó pero no logró inhibir completamente el enranciamiento enzimático que se da naturalmente en la avena.

En el caso del sorgo y la soja el tratamiento de extrusión no presenta diferencias con el tratamiento de molienda tradicional.

En el caso del germen el tratamiento de extrusión tuvo un efecto favorecedor del enranciamiento, probablemente dado por las condiciones del proceso de producción de la harina hayan favorecido la activación de las reacciones del enranciamiento no enzimático oxidativo. A pesar de no haber encontrado reportes de seguimiento de degradación lipídica por oxidación del germen debido al proceso de extrusión, los autores Manuel Gómez, Jesús González y Bonastre Oliete (2012) han logrado resultados positivos en la incorporación del germen de trigo con extrusión en productos panificados. Será necesario continuar estudios en la selección de las condiciones operativas durante la elaboración del germen de trigo.

\section{Ensayo de panificación}

Ha sido extensamente reportado el aumento de la absorción de agua de harinas procesadas por extrusión (Karkle, 2012; Brennan, 2011). En la Tabla 6 se incluyen los valores medios y la mínima diferencia significativa de cada harina. Ensayos previos de panificación indicaron la necesidad de pre-hidratar las harinas antes de usarlas para la elaboración del pan. En el caso de la harina compuesta extrudida se pre-hidrató 1:1. Debido a que la harina compuesta no extrudida absorbe un 40\% menos de agua, se pre-hidrataron en una relación 1:0.6. 


\begin{tabular}{|c|c|c|c|}
\hline & \multicolumn{3}{|c|}{$\begin{array}{l}\text { Índice de absorción de agua } \\
\text { (gramos agua / gramos harina) }\end{array}$} \\
\hline & $\begin{array}{c}\text { Harinas con } \\
\text { extrusión }\end{array}$ & $\begin{array}{c}\text { Harinas sin } \\
\text { extrusión }\end{array}$ & $\begin{array}{c}\text { Pvalor } \\
\text { DMS }\end{array}$ \\
\hline Avena & $5,28 \mathrm{a}$ & $2,84 \mathrm{~b}$ & $\begin{array}{c}\mathbf{0 , 0 0 0 1} \\
0,70\end{array}$ \\
\hline Salvado & $5,56 \mathrm{a}$ & $5,17 \mathrm{~b}$ & $\begin{array}{l}\mathbf{0 , 0 2} \\
0,33\end{array}$ \\
\hline Soja & $5,10 \mathrm{a}$ & $3,70 \mathrm{~b}$ & $\begin{array}{c}\mathbf{0 , 0 0 0 1} \\
0,17\end{array}$ \\
\hline Sorgo & $4,40 \mathrm{a}$ & $2,31 \mathrm{~b}$ & $\begin{array}{c}\mathbf{0 , 0 0 0 1} \\
0,46\end{array}$ \\
\hline $\begin{array}{c}\text { Harina } \\
\text { Compuesta }\end{array}$ & 6,06 & 4,07 & $\begin{array}{c}\mathbf{0 , 0 0 0 1} \\
0,24\end{array}$ \\
\hline
\end{tabular}

Tabla 6. Valor medio, Pvalor y DMS del índice de absorción de las harinas con y sin extrusión. Letras iguales entre valores para un mismo tipo de harina indican que no existen diferencias significativas entre la harina extrudida y no extrudida, según Tukey al $5 \%$.

Los resultados en las harinas sin extrusión de ácidos grasos expresados en $\mathrm{g}$ de oleico/g materia grasa (34,6 en sorgo; 45,1 en avena; 5,2 en soja; 33,6 en salvado) y de humedad expresados en $\mathrm{g}$ de agua/100 g harina (14,0 en sorgo; 11,6 en avena; 10,6 en soja; 14,0 en salvado), previos a la elaboración del pan, fueron similares a los medidos en el día cero del estudio de estabilidad. Sin embargo, el pan elaborado con un $36 \%$ de incorporación de la harina compuesta sin extrusión se vio severamente afectado. Se observó una significativa disminución del volumen, aumento de la dureza, pérdida de elasticidad, aumento de cohesividad y masticabilidad. En la Tabla 7 se presentan los resultados.

\begin{tabular}{|c|c|c|c|}
\hline & \multicolumn{3}{|c|}{ Medidas físicas en pan de molde } \\
\hline & $\begin{array}{l}\text { Pan elaborado con } \\
\text { harinas sin } \\
\text { extrusión }\end{array}$ & $\begin{array}{l}\text { Pan elaborado con } \\
\text { harinas con } \\
\text { extrusión }\end{array}$ & $\begin{array}{c}\text { P - valor } \\
\text { DMS }\end{array}$ \\
\hline Volumen & $431 \mathrm{~b}$ & $545 \mathrm{a}$ & 0,001 \\
\hline Dureza & $1016 \mathrm{a}$ & $551 \mathrm{~b}$ & $\begin{array}{c}\mathbf{0 , 0 0 1} \\
64\end{array}$ \\
\hline Elasticidad & $0,94 \mathrm{a}$ & $0,76 \mathrm{~b}$ & $\begin{array}{c}\mathbf{0 , 0 0 1} \\
0,02\end{array}$ \\
\hline Cohesividad & $0,69 \mathrm{a}$ & $0,50 \mathrm{~b}$ & $\begin{array}{c}\mathbf{0 , 0 0 1} \\
0,01\end{array}$ \\
\hline Masticabilidad & 4697 a & $4192 \mathrm{~b}$ & $\begin{array}{c}\mathbf{0 , 0 0 6} \\
359\end{array}$ \\
\hline
\end{tabular}

Tabla 7. Medidas físicas de pan de molde elaborado con harinas con/sin extrusión. Letras iguales entre valores para un mismo tipo de harina indican que no existen diferencias significativas entre la harina extrudida y no extrudida, según Tukey al $5 \%$.

El pan elaborado con la harina compuesta sin extrusión no fue aceptable por parte de los consumidores, quienes lo describieron como seco y con sabor extraño. Los panes elaborados agregando las harinas con extrusión fueron muy bien aceptados por los consumidores.

\begin{tabular}{|c|c|c|}
\hline Tratamiento & Aceptabilidad & $\begin{array}{c}\text { p-valor } \\
\text { DMS }\end{array}$ \\
\hline Con extrusión & $6,2^{\mathrm{a}}$ & $<\mathbf{0 , 0 0 0 1}$ \\
\hline Sin extrusión & $4,7^{\mathrm{b}}$ & 1,54 \\
\hline
\end{tabular}

Tabla 8. Medida de aceptabilidad sensorial por consumidores de la rodaja de pan elaborado con harinas compuestas con/sin extrusión. Letras distintas dentro de una misma columna indican diferencia significativa en la aceptabilidad $(p>0,05)$.

\section{Conclusión}

El proceso de extrusión disminuye significativamente la velocidad de deterioro de la materia grasa en las harinas de salvado, avena, sorgo y soja. En la harina de germen las condiciones de extrusión ensayadas provocaron un aumento del enranciamiento, limitando su incorporación en alimentos.

El pan de molde con un 36\% de incorporación de harina compuesta extrudida fue sensorialmente aceptable. La incorporación de las harinas sin extrusión dio lugar a un pan sensorialmente no aceptable, con impacto negativo en la textura y el volumen.

\section{Referencias}

- AACC INTERNATIONAL. Approved methods of analysis. 10a ed. Minessota: AACC Internacional, 1999. Method 44-40.

- AACC INTERNATIONAL. Approved methods of analysis. 10a ed. Minessota: AACC Internacional, 1999. Method 46-30.01.

- ANDERSON, R. A.; CONWAY, H. F.; PFEIFER, V. F.; GRIFFIN, E. L. JR. Roll and extrusion cooling of grain sorghum grits'. En: Cereal Science Today. 1969, 14(11):372-376.

- AMERICAN OIL CHEMIST'S SOCIETY. Official method and recommended practices of the AOCS. 6a. Ed. Urbana: AOCS, 2009. Official Method Bc 2-49.

- AMERICAN OIL CHEMIST'S SOCIETY. Official method and recommended practices of the AOCS. 6a. Ed. Urbana: AOCS, 2009. Official Method Bc 9-58.

- AMERICAN OIL CHEMIST'S SOCIETY. Official method and recommended practices of the AOCS. 6a. Ed. Illinois: AOCS, 1989. Official Method Cg 2-83.

- AMERICAN OIL CHEMIST'S SOCIETY. Official method and recommended practices of the AOCS. 6a. ed. Urbana: AOCS, 2009. Official Method Bc 3-49.

- AOAC INTERNATIONAL. Official methods of analysis of AOAC International. 18a. Ed. AOAC, 2010. Official Method 985.29.

- ASAMBLEA MUNDIAL DE LA SALUD. Informe sobre la salud en el mundo: reducir los riesgos y promover una vida sana [En línea]. Ginebra: OMS, 2002. [Consulta : 28 de febrero de 2013]. Disponible en: http://www.who.int/whr/2002/en/whr02_es.pdf

- ATHAR, N.; HARDACRE, A.; TAYLOR, G.; CLARK, S.; HARDING, R.; MCLAUGHLIN, J. Vitamin retention in extruded food products. En: Journal of Food Composition and Analysis. 2006, 19:379-383.

- BRENNAN, CH.; BRENNAN, M.; DERBYSHIRE, E.; TIWARI, B.K. Effects of extrusion on the polyphenols, vitamins and antioxidant activity of foods. En: Trends in Food Science Technology. 2011, 22(10):570-575.

- DOBLADO-MALDONADO, A. F.; PIKE, O.A.; SWELEY, J. C.; ROSE, D. J. Key issues and challenges in whole wheat flour milling and storage. En: Journal of Cereal Science. 2012, 56:119-126.

- GÓMEZ, M.; GONZÁLEZ, J.; OLIETE, B. Effect of extruded wheat germ on dough rheology and bread quality. En: Food Bioprocess Technology. 2012, 5:2409-2418.

- HSU, C. L.; Chen, W.; Weng Y. M.; Tseng, C. Y. Chemical composition, physical properties, and antioxidant activities of yam flours as affected by different drying methods. En: Food Chemistry. 2003, 83:85-92.

- HANSEN, L.; ROSE, M. S. Sensory acceptability is inversely related to development of fat rancidity in bread made from stored flour. En: $J . A m$ Diet. Assoc. 1996, 96:792-793.

- INTERNATIONAL DAIRY FEDERATION (Bélgica). FIL-IDF 99C : 
Sensory evaluation of dairy products by scoring. Bruselas: IDF, 1997.

- INTERNATIONAL ORGANIZATION FOR STANDARDIZATION

(Suiza). ISO 2171: Cereales, legumbres y subproductos. Determinación del rendimiento de cenizas por incineración. Ginebra: ISO, 2010.

- INTERNATIONAL ORGANIZATION FOR STANDARDIZATION

(Suiza). ISO 8589: Sensory analysis - General guidance of the design of test room. Ginebra: ISO, 2007.

- INSTITUTO ARGENTINO DE NORMALIZACIÓN Y

CERTIFICACION (Argentina). IRAM 20005-1: Análisis sensorial.

Guía general para la selección, entrenamiento y seguimiento de los evaluadores. Parte 1: evaluadores seleccionados. Buenos Aires: IRAM, 1996.

- KARKLE, E.; KELLER, L.; DOGAN, H.; ALAVI, S. Matrix transformation in fiber-added extruded products: impact of different hydration regimens on texture, microstructure and digestibility. En: Journal of Food Engineering. 2012, 108:171-182.

- KILLEIT, U. Vitamin retention in extrusion cooking. En: Food Chemistry. 1994, 49:149-155.

- POMERANZ, Y. Biochemical, functional, and nutritive changes during storage. En: SAUER, D. B. Storage of cereal grains and their products. 4ta. ed. Minnesota: AACC International, 1992. pp. 55-141.

- ROSE, D. J.; OGDEN, L.; DUNN, M.L.; PIKE, O.A. Enhanced lipid stability in whole wheat flour by lipase inactivation and antioxidant retention. En: Cereal Chemistry. 2008, 2:218-223.

- SOSA MOGUEL, O.; RUIZ-RUIZ, J.; MARTINEZ-AYALA, A.; GONZALEZ, R.; DRAGO, S.; BETANCUR-ANCONA, D.; CHELGUERRERO, L. Effect of extrusion conditions and lipxygenase inactivation treatment on physical and nutritional properties of corn/ cowpea (Vigna unguiculata) blends. En: International Journal of Food Sciences and Nutrition. 2009 60(S7):341-354.

- TAIT, S.P.; GALLIARD, T. Effect of baking quality of changes in lipid composition during wholemeal storage. En: Journal of Cereal Science. 1988, 8:125-137.

- TURGEON, S.; RIOUX, L. Food matrix impact on macronutrients nutritional properties. En: Food Hydrocolloids. 2011, 25:1915-1924.

- U.S. DEPARTMENT OF AGRICULTURE; U.S. DEPARTMENT OF HEALTH AND HUMAN SERVICES. Dietary guidelines for americans. $7 \mathrm{ma}$. ed. Washington: U.S. Government Printing Office, 2010. pp.29-30.

- U.S. FOOD AND DRUG ADMINISTRATION. Health claim notification for whole grain food with moderate fat content [En línea]. Silver Spring: FDA, 2003. [Consulta: 28 de febrero de 2013]. Disponible en: http://www.fda.gov/Food/IngredientsPackagingLabeling/ LabelingNutrition/ucm073634.htm

- VETRIMANI, R.; HARIDAS, R. Studies on stabilization of wheat bran. En: Journal of Food Science and Technology. 1990, 27:332-335. 